\title{
A solid-state phase conjugate mirror for space Lidar systems
}

\author{
A. Brignon ', S. Richard ${ }^{1}$, M. Georges ${ }^{2}$, J-Y. Plesseria ${ }^{2}$, T. Thibert ${ }^{2}$, P-A. Blanche ${ }^{2}$, A. Gusarov ${ }^{3}$, F. Berghmans ${ }^{3}$, \\ Y. Lien $^{4}$ \\ 1. Thales Research \& Technology, RD 128, 91767 Palaiseau cedex, France \\ 2. Centre Spatial de Liège, Université de Liège, Liege Science Park, B-4031 Angleur-Liège, Belgium \\ 3. Studiecentrum voor Kernenergie, Centre d'Etude de l'Energie Nucléaire, Boeretang 200, B-2400 Mol, Belgium \\ 4. European Space Agency, ESTEC, TEC-MME, Postbus 299, 2200 AG Noordwijk, The Netherlands
}

Overall system performance in pulsed Lidars strongly depends on the spatial quality of the emitted beam and the attained pulse energy level. It becomes increasingly difficult to maintain a good beam quality with increasing output powers or pulse energies required by future operational systems. Transmitter configurations employing wavefront corrections are thus needed. Virtually all commercially available phase conjugate mirror (PCM) are realized in a liquid or gaseous cell using stimulated Brillouin Scattering (SBS). Their chemical aggressiveness with the associated difficulty in handling makes these cells rather unsuitable for space use. Among solid-state materials, fused silica has already shown a good potential at $10 \mathrm{~Hz}$ [1]. In this communication, we present a solid-state PCM based on SBS in a fused silica rod suitable for space environment with reliable operation at higher repetition rates.

Different types of silica have been tested and compared including HOQ 310, Infrasil 301, Suprasil 1, Suprasil 311 and Lithosil. For each material, the SBS response and the laser induced damage (LID) threshold have been studied. Impact of protons and gammarays irradiations on the performances of each type of silica have also been conducted. From this study, we

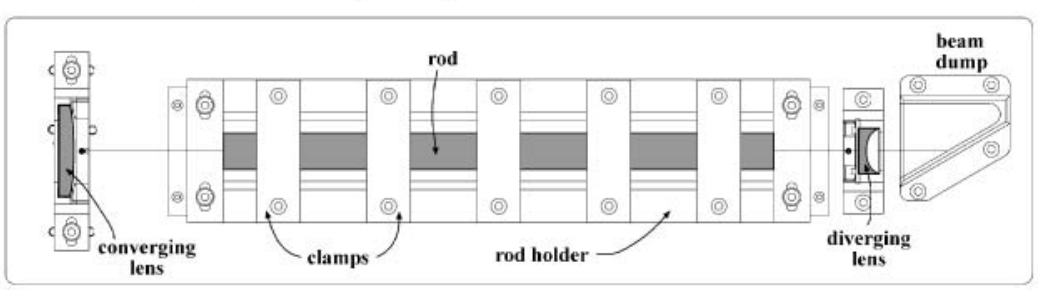

Fig. 1 : schematic of the all-solid-state PCM breadboard. conclude that Lithosil and Suprasil are the best materials for PCM at $1064 \mathrm{~nm}$.

A PCM breadboard compatible with vacuum environment and acceleration up to $50 \mathrm{~g}$ as shown in Figure 1 has been constructed. Phase conjugation test experiments have been conducted with two single frequency flash-lamp pumped $\mathrm{Nd}$ :YAG laser systems. The first one delivering up to $220 \mathrm{~mJ}$ pulses with a duration of $20 \mathrm{~ns}$ at $50 \mathrm{~Hz}$ repetition rate, the second laser delivering up to $200 \mathrm{~mJ}$ at $100 \mathrm{~Hz}$. The PCM is formed by focusing the laser beam inside a $30 \mathrm{~cm}$ long silica rod. The focusing geometry has been optimized to avoid LID at the entrance and exit face of the rod as well as in the bulk of the material. The SBS threshold is observed at about $15 \mathrm{~mJ}$ pulse energy and a phase conjugate reflectivity up to $80 \%$ has been obatined with $220 \mathrm{~mJ}$ incident pulse energy. Under this condition, stable operation of the PCM has been observed for more than $2 \times 10^{5}$ pulses at $50 \mathrm{~Hz}$ pulse repetition rate. Reliable operation at $200 \mathrm{~mJ}, 100 \mathrm{~Hz}$ has been also demonstrated.

Vacuum-thermal tests have been also conducted. It is shown that the performances of the PCM are not altered when it is used under vacuum (typically $10^{-5}$ mbar) and for temperatures ranging between $0^{\circ}$ to $30^{\circ} \mathrm{C}$. The mechanical concept proposed is viable and does not provide stresses that endanger the integrity of optical elements when undergoing temperature going from $-60^{\circ} \mathrm{C}$ to $+60^{\circ} \mathrm{C}$. Finally, the performances of the PCM are not affected after irradiation by both protons and gamma-rays.

To conclude, we have developped a reliable spacequalifiable solid-state phase conjugate mirror operating up to $220 \mathrm{~mJ}$ with reflectivity of $80 \%$. It is expected that such a component will permit a significant increase of the beam quality of Lidar laser systems under development for space applications.

\section{References}

1. H. Yoshida, and M. Nakatsuka, "Advanced stimulated Brillouin Scattering for Phase Conjugate Mirror using LAP, DLAP, and silica glass", in : A. Brignon and J.-P. Huignard (eds.), Phase Conjugate Laser Optics, Wiley (2004)

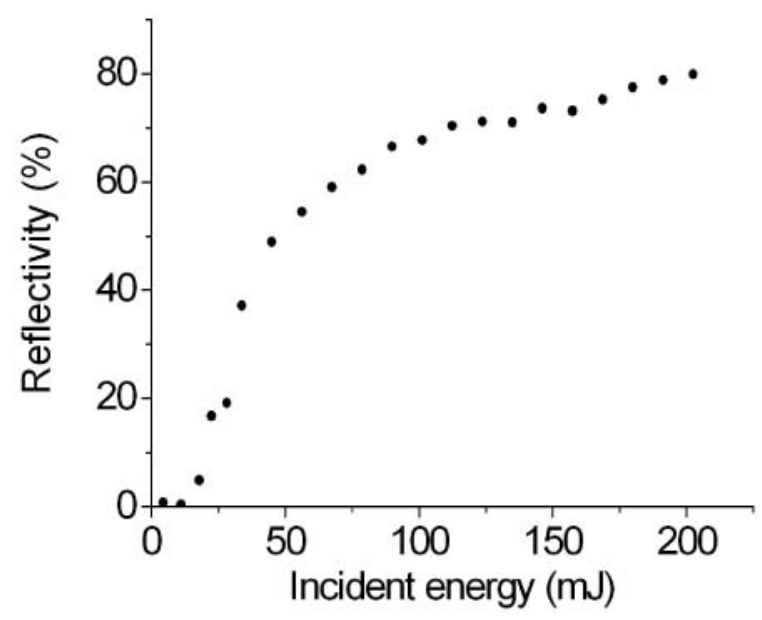

Fig. 2 : phase conjugate reflectivity as a function of the incident pulse energy. 